\title{
Quantitative ultrashort echo time magnetization transfer (UTE-MT) for diagnosis of early cartilage degeneration: comparison with UTE-T2* and T2 mapping
}

\author{
Jiawei Yang ${ }^{1 \#}$, Hongda Shao ${ }^{1 \#}$, Yajun $\mathrm{Ma}^{2}$, Lidi Wan ${ }^{1}$, Yixuan Zhang ${ }^{1}$, Junjie Jiang ${ }^{3}$, Jiang $\mathrm{Du}^{2}$, \\ Guangyu Tang
}

${ }^{1}$ Department of Radiology, Shanghai Tenth People's Hospital, Tongji University School of Medicine, Shanghai 200072, China; ${ }^{2}$ Department of Radiology, University of California, San Diego, CA, USA; ${ }^{3}$ Department of Orthopedics, Shanghai Tenth People's Hospital, Tongji University School of Medicine, Shanghai 200072, China

\#These authors contributed equally to this work.

Correspondence to: Guangyu Tang, PhD. Department of Radiology, Shanghai Tenth People's Hospital, Tongji University School of Medicine, 301 Middle Yanchang Road, Shanghai 200072, China. Email: tgy17@126.com.

Background: To investigate the feasibility of using quantitative ultrashort echo time magnetization transfer (UTE-MT) technique in diagnosing early cartilage degeneration and to compare the technique's diagnostic efficacy with UTE-T2* mapping and T2 mapping.

Methods: Twenty human anterolateral condyle specimens with degeneration were obtained from volunteers undergoing total knee arthroplasty (TKA); they then underwent magnetic resonance (MR) scan on a clinical 3.0T scanner (GE, MR750). Seventy-two regions of interest (ROI) were manually drawn on specimens for UTE-MT, UTE-T2*, and T2 measurement, and the corresponding cartilage-bone regions were further divided into degeneration classifications of normal ( $\mathrm{n}=11$, Mankin scores $0-1)$, mild ( $\mathrm{n}=28$, Mankin scores 2-5), moderate ( $n=21$, Mankin scores 6-9), and severe ( $n=12$, Mankin scores 10-14) based on histological measures of degeneration (i.e., Mankin scores) as a reference standard. Differences among groups and correlations between quantitative MR parameters and Mankin scores were assessed using analysis of variance (ANOVA), Tamhane-T2, LSD, Kruskal-Wallis tests, and Spearman's correlation coefficient. The receiveroperating characteristic (ROC) curve was used to compare the diagnostic efficacy of different quantitative MR parameters for the detection of mild cartilage degeneration.

Results: The UTE magnetization transfer ratio (UTE-MTR) in the normal group was significantly different from the mild group $(\mathrm{P}=0.021)$, moderate group $(\mathrm{P}<0.001)$, and severe group $(\mathrm{P}<0.001)$. Significant differences were observed in the $\mathrm{T} 2$ * values between both the normal group and the moderate group $(\mathrm{P}<0.032)$, and between the normal group and the severe group $(\mathrm{P}<0.001)$. For $\mathrm{T} 2$ values, the only significant difference was observed between the severe group and the normal group $(\mathrm{P}=0.011)$. The UTE-MTR, UTE-T2*, and T2 values were all significantly correlated with Mankin scores: UTE-MTR values were strongly $(\mathrm{r}=-0.678, \mathrm{P}<0.001)$ correlated, UTE-T2* values were markedly correlated $(\mathrm{r}=-0.501, \mathrm{P}<0.001)$, and $\mathrm{T} 2$ values were weakly correlated $(\mathrm{r}=0.337, \mathrm{P}=0.004)$ correlated with Mankin scores. The diagnostic efficacy of UTE-MTR (AUC =0.828, P=0.002) was better than UTE T2* mapping and T2 mapping (AUC =0.604, $\mathrm{P}=0.318$; $\mathrm{AUC}=0.644, \mathrm{P}=0.165$, respectively) for the diagnosis of early cartilage degeneration.

Conclusions: UTE-MTR values were strongly correlated with histological grades of cartilage degeneration, and its diagnostic efficacy was better than both UTE T2* mapping and T2 mapping in detecting early cartilage degeneration. Once the clinical potential of the technique has been confirmed, UTE-MT may provide a promising imaging biomarker with potential application in a more comprehensive diagnosis and monitoring of cartilage degeneration. 
Keywords: Magnetic resonance imaging (MRI); ultrashort echo time magnetization transfer (UTE-MT); magnetization transfer ratio (MTR); cartilage degeneration; early diagnosis

Submitted Aug 09, 2019. Accepted for publication Nov 28, 2019.

doi: 10.21037/qims.2019.12.04

View this article at: http://dx.doi.org/10.21037/qims.2019.12.04

\section{Introduction}

Osteoarthritis (OA) is one of the most common diseases of the human joints and features progressive degeneration of articular cartilage, which severely affects the quality of life, work productivity, and healthcare costs (1-3). The onset of $\mathrm{OA}$ is characterized primarily by the loss of proteoglycan (PG) and the destruction of collagen fibers in articular cartilage, with PG depletion being reversible (4). Currently, clinical imaging techniques [e.g., X-ray, computed tomography (CT), and conventional magnetic resonance imaging (MRI)] can only detect the morphological changes of OA in its advanced stages, namely the formation of osteophytes, the sclerosis of subchondral bone, and narrowing of the joint space, which are all irreversible (5-7). Imaging techniques that are sensitive to the subtle changes of biochemical components evident in sub-clinical OA at its early stages are of primary importance in improving the diagnosis, treatment, and monitoring of this disease.

Conventional quantitative cartilage imaging techniques, such as T2/T2* mapping and T1rho mapping, allow for direct, non-invasive assessment of both matrix alterations and the loading dynamics of articular cartilage which can precede morphologic changes visible on clinical sequences (8-16). The degenerative cartilage processes that disrupt the structure of the cartilage matrix impact $\mathrm{T} 2 / \mathrm{T} 2 *$ relaxation time. Although the common consensus generally asserts that $\mathrm{T} 2 / \mathrm{T} 2 *$ mapping is sensitive to cartilage matrix hydration and the orientation of collagen within the matrix $(17,18)$, there are other factors impacting T2/T2* values which require further investigation (13-15). Another primary quantitative cartilage imaging biomarker, T1rho, reflects the low-magnitude movements and interactions between the constrained water protons and their local environment. Some studies have reported that T1rho value has a strong correlation with the PG loss that precedes the onset of cartilage degeneration $(19,20)$ in $\mathrm{OA}$, while other studies have found additional factors such as tissue hydration and collagen orientation to be contributors as well $(14,21)$.

In recent years, conventional quantitative MRI techniques (e.g., T2/T2* mapping and T1rho mapping) have demonstrated considerable inter- and intra-individual variability, as well as susceptibility to the magic angle effect, which makes the differentiation of early-to-moderate stages of cartilage degeneration challenging (22). When the fibers of the cartilage matrix are oriented near the magic angle, $\mathrm{T} 2$ values show an increase of $231.8 \%$, and T1rho values show an increase of $92 \%$, regardless of whether the cartilage is normal or abnormal (23). Furthermore, conventional quantitative MRI techniques such as T1rho and T2 mapping are useful in depicting changes to the transitional and superficial cartilage layers (24) where relatively long T2 relaxations ( $>10 \mathrm{~ms}$ ) typically dominate, but these techniques are not sensitive to minor alterations in the deep radial and calcified cartilage, which have very short $\mathrm{T} 2$ relaxation times $(<10 \mathrm{~ms})(25)$. Increased stress in the tissue may lead to early matrix deterioration of the cartilage (26-28).

$3 \mathrm{D}$ ultrashort echo time (UTE) sequencing is a novel MRI technique that, when combined with several conventional imaging sequences, is sensitive to the short $\mathrm{T} 2$ signal of articular cartilage and is less sensitive to the magic angle effect (28-32). Our previous studies (30) have shown that the 3D UTE sequence combined with magnetization transfer (3D UTE-MT-Cones) analyzed by UTE-MT two pool modeling measurements can generate unique contrast and quantitative information on articular cartilage due to the coupling processes between macromolecular and mobile protons of a cartilaginous matrix. However, to the best of our knowledge, studies on UTE-MT quantitative technique in evaluating articular cartilage degeneration are scarce.

This study aimed to investigate the feasibility of quantitative UTE-MT technique in the diagnosis of early cartilage degeneration and compare the technique's diagnostic efficacies with those of UTE-T2* and T2 mapping. Our hypothesis was that UTE-MTR values would correlate well with the histological grades of degeneration in human articular cartilage, thereby facilitating the differentiation of early degeneration in cartilage for improved disease diagnosis. 


\section{Methods}

\section{Human anterolateral femoral condyle procurement}

Twenty fresh human anterolateral femoral condyles were obtained from 20 donors with primary $\mathrm{OA}$ who underwent total knee arthroplasty (TKA) at our research hospital, 11 of whom were male (49-87 years; $60.4 \pm 18.2$ years old), and 9 of whom were female (52-90 years; $62.3 \pm 19.3$ years old). Informed consent was obtained from each donor before surgery (Ethics Committee approval number: shsy-iecky-3964).

Any donor who had underlying neoplastic or rheumatologic diseases, or a history of trauma, fracture, or previous surgery of the knee joint was excluded. For the sake of topoanatomic consistency, samples were only harvested from the anterolateral femoral condyles. The anterolateral femoral condyle was chosen since it generally showed moderate OA-related cartilage degeneration compared to the weight-bearing region of the lateral femoral condyle. After harvest, the surrounding soft tissue was removed. A transverse slab of a 4-mm thickness (sagittal direction) was obtained from the center of each anterolateral femoral condyle specimen and embedded in gauze soaked with phosphate-buffered saline (PBS), then stored at $-80^{\circ} \mathrm{C}$.

\section{MR data acquisition}

The specimens were removed from the $-80{ }^{\circ} \mathrm{C}$ refrigerator and defrosted at room temperature for 24 hours before the MR scan, which was performed on a clinical 3.0 T MRI scanner (MR750, GE Healthcare, Waukesha, WI, USA). Susceptibility effects at the tissue-air junction during scanning were minimized by soaking the specimens in Fomblin and placing them at the isocenter of the MR scanner. UTEMT (off-resonance frequency $=2 \mathrm{KHZ}$, saturation power $=750 \mathrm{KHZ}, \mathrm{TR} / \mathrm{TE}=100 / 0.032 \mathrm{~ms}$; scan time $=1: 48 \mathrm{~min}$ ), UTE-T2* mapping [TEs $=0.032,4.9,9.8$, and $14.7 \mathrm{~ms}$; repetition time $(\mathrm{TR})=33 \mathrm{~ms}$; scan time $=5: 43 \mathrm{~min}]$, and conventional T2 mapping sequences (TEs $=6,18,25$, and $38 \mathrm{~ms}$; TR $=1,285 \mathrm{~ms}$; scan time $=2: 05 \mathrm{~min}$ ) were performed for UTE-MTR, T2*, and T2 measurement. Other imaging parameters were as follows: field of view $(\mathrm{FOV})=8 \mathrm{~cm}$, acquisition matrix $=256 \times 256$ pixels, slice thickness $=2 \mathrm{~mm}$. The pixel size was $0.3125 \times 0.3125 \mathrm{~mm} /$ voxel for all images.

\section{Postprocessing and image analysis}

Each image was divided into 4 generally equal regions, within which 4 regions of interest (ROIs) were manually drawn. These ROIs were precisely matched to mid-sagittal histology images of each specimen slab, as shown in Figure 1. ROIs of each specimen were manually drawn on the middle UTE image of each series and then copied to each of the other series. Eight ROIs were excluded since the cartilage was almost entirely absent due to severe degeneration. The remaining 72 ROIs had approximately $79.4 \pm 22.2$ [mean \pm standard deviation (SD)] pixels per ROI. All quantitative MR parameters were measured by one author who had a special interest in musculoskeletal radiology and was blinded to the histological assessment. Quantitative UTE image analysis algorithms were performed in MatLab (Mathworks Inc., Natick, MA, USA) using the LevenbergMarquardt method for nonlinear least-squares curve fitting, and analysis was executed offline on DICOM images obtained by the protocols described above. The mean intensity within ROI each was used for curve fitting. Single-component fitting models acquired from the dualecho 3D-UTE-Cones sequence were utilized for T2* decay analyses. UTE-MTR was acquired with a set of MT pulse frequency offsets $(2 \mathrm{KHz})$ and powers $(0 / 750 \mathrm{KHZ})$ by twopool MT modeling. The measurement of spin echo (SE)based $\mathrm{T} 2$ values was performed on a GE workstation using T2 imaging software. Each ROI was drawn repeatedly in the same region 3 times, and the average signal intensity was used for statistical analysis.

\section{Tissue processing}

After MRI scanning, the specimens were immediately fixed in Z-Fix (Anatech, Battle Creek, MI, USA) for 3 days, then decalcified with EDTA (Thermo Scientific, Kalamazoo, MI, USA). Accurate orientation was ensured by selecting the central level of each sample and labeling it with a tissue-marking dye (Cancer Diagnostics, Morrisville, NC, USA). Each sample block was trimmed on a microtome after adequate decalcification, dehydration with alcohol, and infiltration with paraffin. A 3- $\mathrm{m}$ cross-section from the center of each sample, including the cartilage and subchondral bone, was cut to match the MRI scans. Then, the samples were stained with Safranin O-Fast Green according to standard protocols $(33,34)$ for histopathologic analysis.

\section{Histopathology analysis}

All Safranin O-Fast Green-stained slides were viewed 

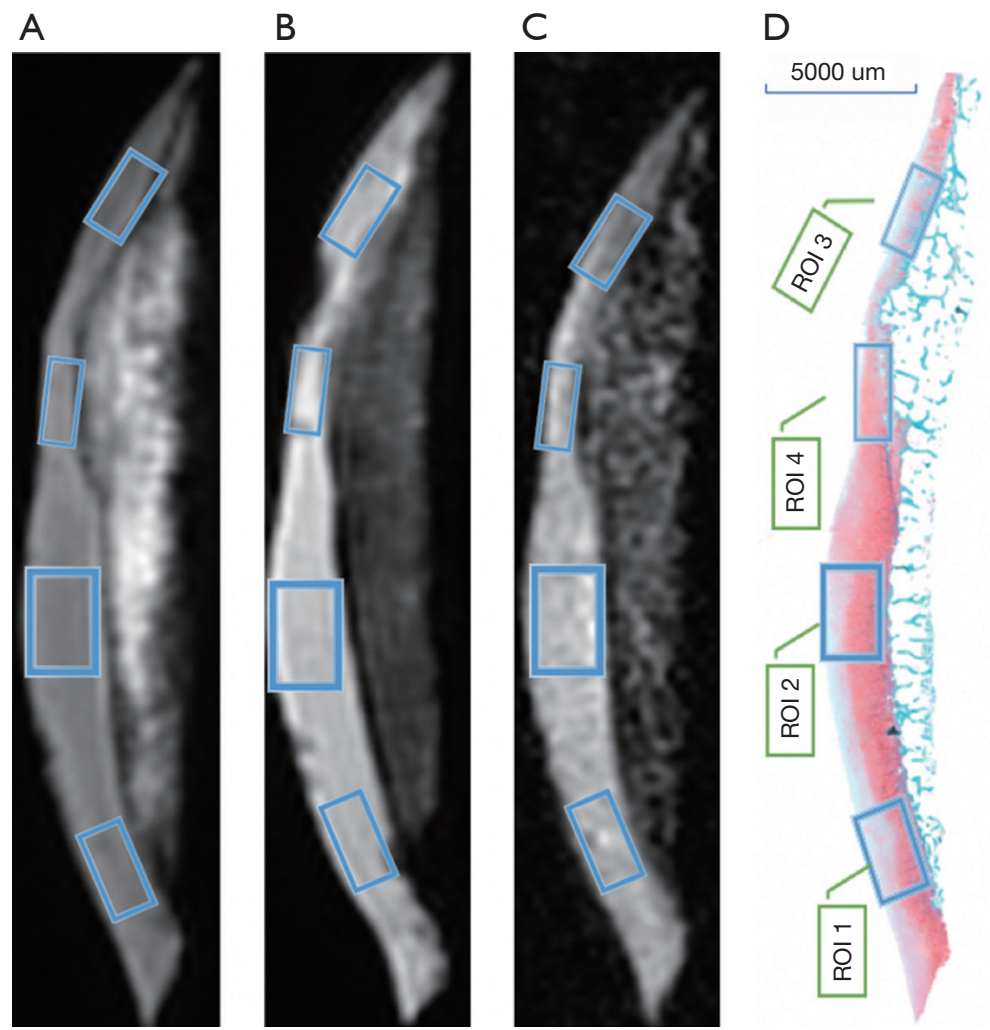

Figure 1 Four different ROIs from a human anterolateral femoral condyle sample. (A) UTE-MT image; (B) UTE-T2* mapping image; (C) T2 mapping image; (D) Safranin O-Fast Green staining image of the corresponding specimen; ROI 1 with mild degeneration (Mankin score 4), ROI 2 with mild degeneration (Mankin score 3), ROI 1 with severe degeneration (Mankin score 12), and ROI 4 with severe degeneration (Mankin score 11). ROIs, regions of interest; UTE-MT, ultrashort echo time magnetization transfer.

with a Nikon E100 slide scanner and scanned with SlidePath software (Nikon Microsystems, Tokyo, Japan). The cross-sections of each sample most likely covered several regions with different histopathologic grades, with the goal of thoroughly covering all possible grades within each specimen. The corresponding ROIs of the MR image was selected for histopathologic correlation and further divided into 4 groups (i.e., normal, mild, moderate, and severe degeneration groups, presented as groups 1, 2, 3, and 4 , respectively) using Mankin scores as a reference standard (35). Each ROI was evaluated by a pathologist with 10 years of musculoskeletal research experience who assigned a Mankin score ranging from 0 to 14 . Each score was converted to a grade as follows: grade 1 for scores $0-1$, grade 2 for scores 2-5, grade 3 for scores 6-9, and grade 4 for scores 10-14. Grade 1 represented normal tissue; grade 2 , mild degeneration; grade 3, moderate degeneration; and grade 4 , severe degeneration.

\section{Statistical analysis}

All data analysis was processed with SPSS 24.0 software (SPSS, Chicago, IL, USA). As normal distributions could not be confirmed for T2 values of this study, the mean ${ }_{ \pm} \mathrm{SD}$ or median and interquartile range (IQR) were used to express the variable of normal (UTE-MTR, UTE-T2* values) and non-normal (T2 values) distributions, respectively. One-way ANOVA was used to compare UTEMTR and UTE-T2* mapping values among groups. Tamhane-T2 and LSD tests were used for additional multiple comparisons. The T2 mapping values of different groups were compared with the Kruskal-Wallis test. Spearman's correlation coefficient was used to evaluate the correlation between quantitative MR results and Mankin scores. Receiver-operating characteristic (ROC) curves were assessed to evaluate the diagnostic efficacy of different quantitative biomarkers for detecting early cartilage lesions. Correlations were classified and considered very 
Table 1 Mean or median MRI parameters (MTR, T2*, T2) of four different Mankin grades

\begin{tabular}{lccr}
\hline Mankin grade & MTR\% & T2* ms & T2 ms \\
\hline Grade 1 $(n=11)$ & $16.30 \pm 0.013$ & $20.30 \pm 7.26$ & $41.10(36.60-44.30)$ \\
Grade 2 $(n=28)$ & $14.78 \pm 0.012$ & $18.52 \pm 6.07$ & $42.80(39.40-46.28)$ \\
Grade 3 $(n=21)$ & $13.93 \pm 0.010$ & $15.42 \pm 5.85$ & $42.10(40.00-44.50)$ \\
Grade 4 $(n=12)$ & $12.45 \pm 0.022$ & $10.91 \pm 4.16$ & $49.20(44.38-56.70)$ \\
\hline
\end{tabular}

MRI, magnetic resonance imaging; MTR, magnetization transfer ratio.

strong, strong, marked, low, or negligible with correlation coefficients of $1.0 \geq r>0.80,0.80 \geq r>0.60,0.60 \geq r>0.40$, $0.40 \geq r>0.20$, and $0.20 \geq r$, respectively. $P$ values less than 0.05 were considered statistically significant.

\section{Results}

\section{Comparison of T2 mapping, UTE-T2* mapping, and UTE-MTR among different bistopathologic groups}

The quantitative values of the 72 ROIs areas are presented in Table 1, and the corresponding quantitative MR images are shown in Figure 2. A comparison of T2 mapping, UTE-T2* mapping, and UTE magnetization transfer ratio (MTR) among different histopathologic groups is shown in Figure 3. UTE-MTR values of group 1 were significantly higher than the other groups (group 1 vs. groups 2, 3, or 4; $\mathrm{P}=0.021, \mathrm{P}<0.001$, and $\mathrm{P}<0.001$, respectively). Meanwhile, a significant decrease in MTR values was observed in groups 3 and 4 compared to group $2(\mathrm{P}=0.047$ and $\mathrm{P}=0.026$, respectively). The difference of UTE $\mathrm{T} 2{ }^{*}$ values between groups 1 and 2 was not significant $(\mathrm{P}=0.418)$, whereas the UTE T2* values of group 1 were significantly higher than both groups 3 and $4(\mathrm{P}=0.032$ and $\mathrm{P}<0.001$, respectively). For $\mathrm{T} 2$ values, significant differences were only observed between group 4 and group $1(\mathrm{P}<0.011)$.

\section{Correlation analysis}

The correlation between quantitative MR results and the Mankin scores in the 72 regional ROIs is shown in Figure 4. UTE-MTR and UTE-T2* values were significantly negatively correlated with Mankin scores $(\mathrm{P}<0.001)$, whereas T2 values were positively correlated with Mankin scores $(\mathrm{P}=0.004)$. UTE-MTR values correlated strongly with Mankin scores $(\mathrm{r}=-0.678)$, UTE-T2* correlated markedly $(\mathrm{r}=-0.501)$, and $\mathrm{T} 2$ values correlated weakly $(\mathrm{r}=0.337)$.

\section{Evaluation of the diagnostic value of UTE-MTR, UTE-T2*, and T2 mapping for early degeneration of cartilage by ROC curve analysis}

As shown in Figure 5, the areas under the curve (AUC) of UTE-MTR (AUC $=0.828, \mathrm{P}=0.002$ ) were significantly higher than those of UTE T2* (AUC $=0.604, \mathrm{P}=0.318$ ) and T2 mapping ( $\mathrm{AUC}=0.644, \mathrm{P}=0.165$ ) at 0.05 significance level. Additionally, no significant diagnostic value was found in UTE-T2* and T2 values according to the ROC curves, whereas the diagnostic threshold values of MTR for feasible differentiation of normal cartilage from early degenerative cartilage was $15.5 \%$, and the corresponding sensitivity and specificity values were $81.8 \%$ and $71.4 \%$, respectively. Overall, the diagnostic efficacy of UTE-MTR was better than that of UTE T2* mapping and T2 mapping for the evaluation of early degeneration of articular cartilage.

\section{Discussion}

The most important finding of this study is that UTEMTR values were strongly correlated with histological grades (Mankin scores) of cartilage degeneration and that its diagnostic efficacy was better than that of UTE T2* mapping and T2 mapping in detecting early cartilage degeneration. In the present study, quantitative MR imaging was performed in a clinical-like setup utilizing practicable scanning times to investigate the feasibility of using UTE-MT, UTE T2*, and T2 mapping techniques for the detection of articular cartilage in humans. T2 mapping is now widely used in the evaluation of articular cartilage (36-45). However, to the best of our knowledge, UTEMT has yet to be systematically studied in the evaluation of articular cartilage degeneration. Furthermore, this study was the first to investigate and compare the diagnostic efficacies of UTE-MT, UTE T2*, and T2 mapping in detecting the degeneration of human articular cartilage. Although 

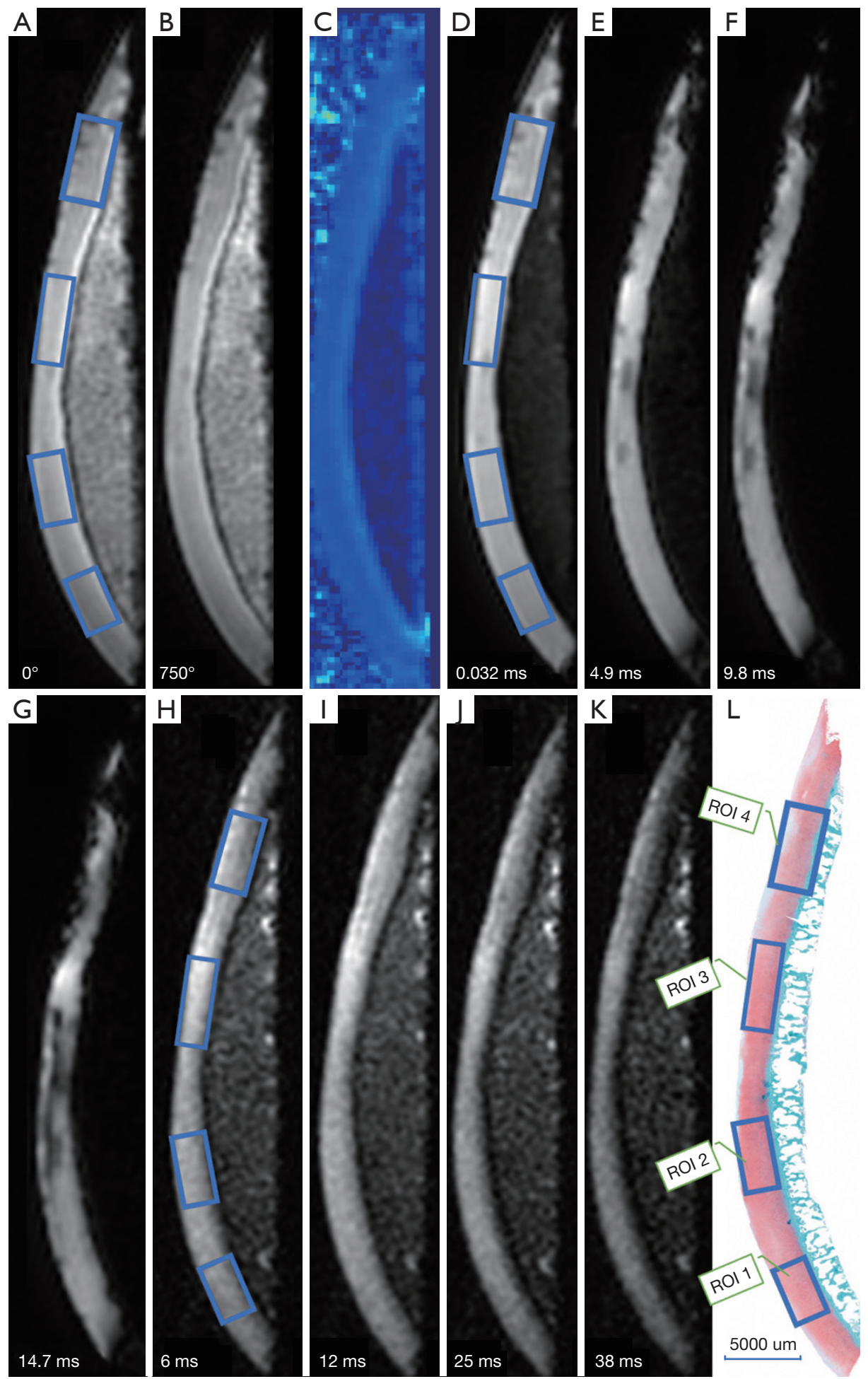

Figure 2 A human anterolateral femoral condyle sample with different MR images and the corresponding Safranin o-fast Green staining image. (A,B) UTE-MT images of a human anterolateral femoral condyle sample with different saturation powers and (C) the corresponding UTE-MT pixel map; (D,E,F,G) UTE-T2* mapping images of a human anterolateral femoral condyle with different TE times; (H,IJ,K) T2 mapping images of a human anterolateral femoral condyle with different TE times; (L) Safranin O-Fast Green staining image of the corresponding specimen. ROIs 1 and 2 are the normal groups (Mankin score $=1$ ), while ROIs 3 and 4 are the moderate degeneration group (Mankin score =3, 5, respectively). ROIs, regions of interest; UTE-MT, ultrashort echo time magnetization transfer. 
A

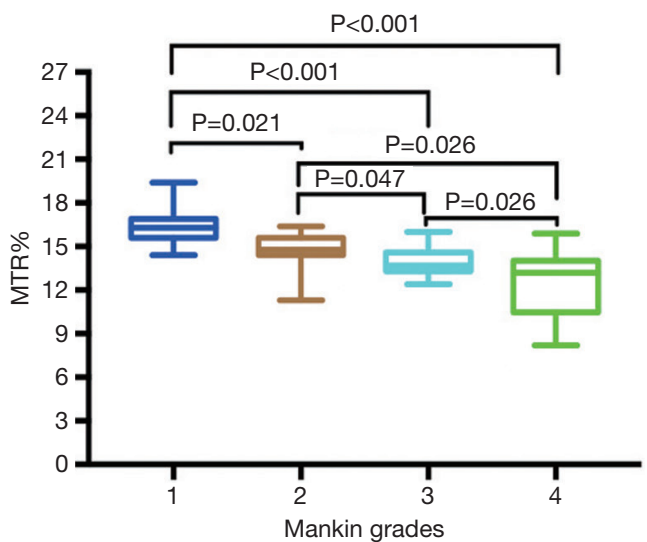

C

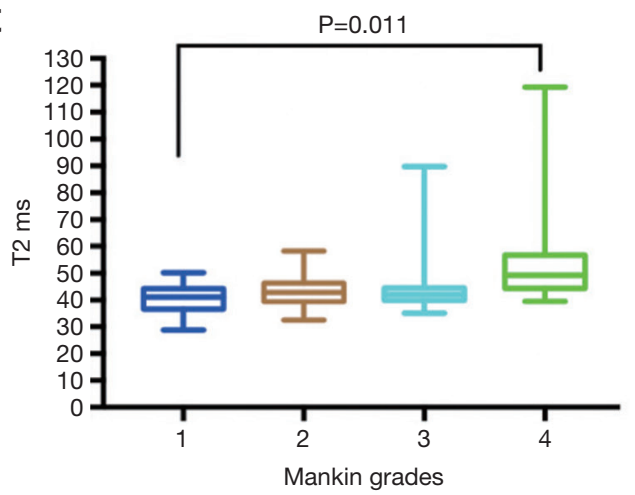

B

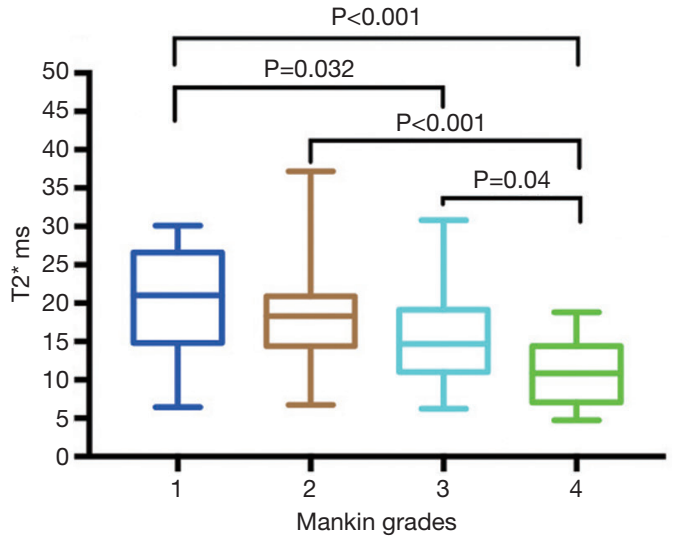

Figure 3 Box-plot diagrams illustrating the MTR, T2*, and T2 values according to the Mankin grades. (A) Tamhane-T2 test used to measure multiple comparisons among different grades. (B) LSD test used to measure multiple comparisons among different grades. (C) Kruskal-Wallis test used to measure multiple comparisons among different grades. MTR, magnetization transfer ratio.

the exact diagnostic value of UTE-MTR in the in vivo evaluation of cartilage still requires further investigation, there is considerable potential to extend our findings into the clinical realm.

The MT imaging technique is currently a valuable tool for obtaining the structure and biochemical composition of cartilage (46-50). MT measures have also been shown to have much-reduced orientation dependence compared with T2/T2* relaxation measurements (51). The standard MTR $=1-\left(M_{s a t} / M_{0}\right)$, where $M_{\text {sat }}$ and $M_{0}$ represent magnetization with and without off-resonance saturation, respectively $(46,47)$. Previous study (48) showed that the MTR value was useful in detecting trypsin-digested and nondigested bovine cartilage, with a decrease in MTR indicating weakened exchange between the free (unbound) water pool and the bound proton pool, suggesting that protons tended to bind with macromolecules (e.g., collagen and PG) during the degeneration of cartilage (48). However, Zuo et al. (49) and Welsch et al. (50) found no significant difference in the conventional MTR values (based on conventional clinical sequences) of cartilage between healthy volunteers and patients with OA, suggesting that MTR values based on conventional clinical sequences may have limited application in the diagnosis of clinical OA. Similarly, a multi-parametric MRI study showed that conventional MTR values were not found to be significantly affected by collagenase treatment in the deep cartilage (52). However, several other $(26,27,30,33)$ studies showed that deep cartilage is an important interface between cartilage and bone since it not only attaches cartilage to the bone but also transmits force between the two surfaces. These studies, therefore, suggest that deep cartilage may play a vital role in the mechanisms underlying early cartilage degeneration. That being said, consistently increasing stress in deep cartilage may lead to the early matrix deterioration of cartilage, resulting in the continuous loss of large macromolecules in the cartilage matrix. Notably, deep cartilage has a very short $\mathrm{T} 2$ relaxation time, rendering it effectively undetectable on 

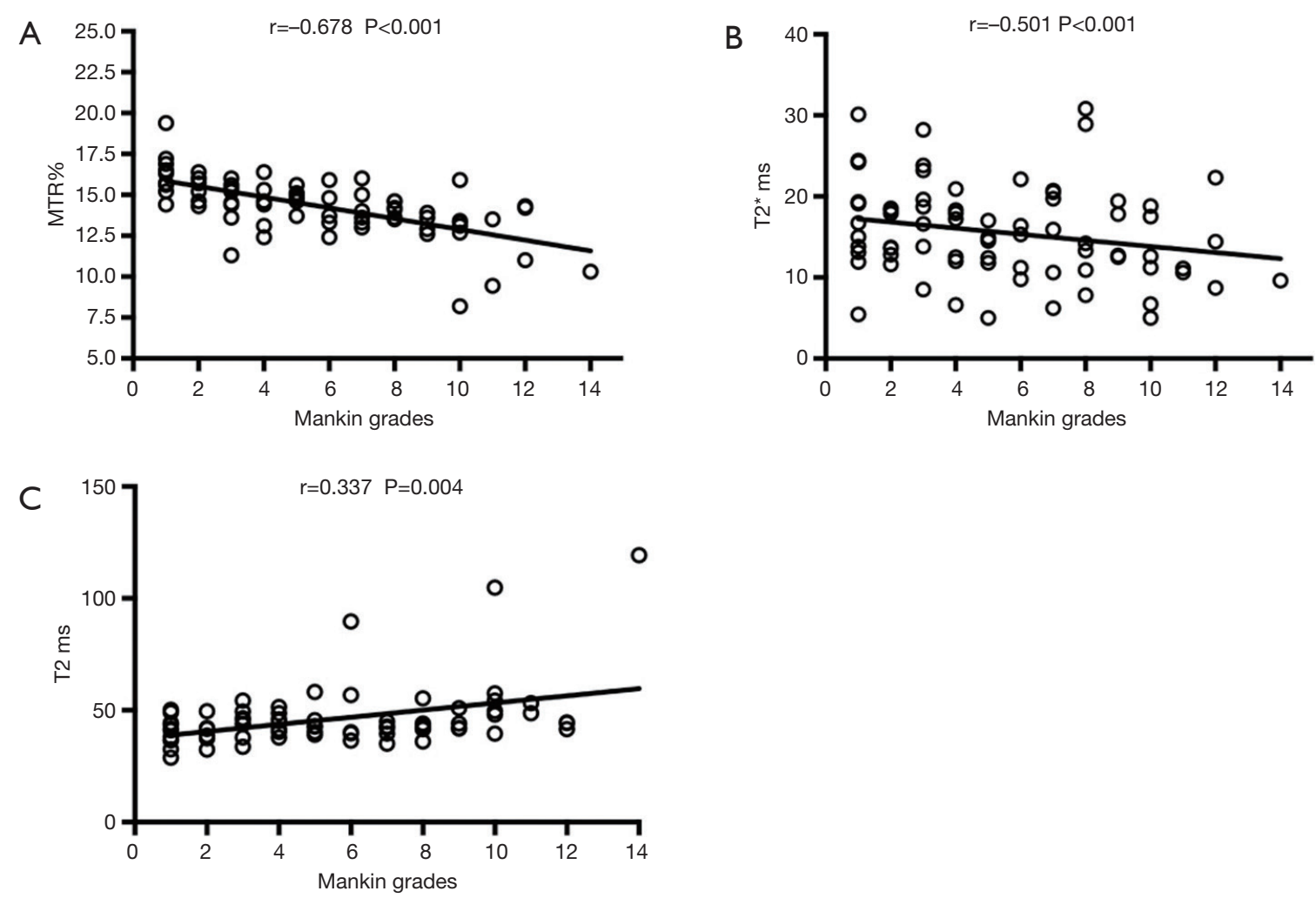

Figure 4 Spearman's correlation between quantitative MR results and the Mankin scores. (A) Spearman's correlation between MTR values and Mankin scores; (B) Spearman's correlation between UTE-T2* values and Mankin scores; (C) Spearman's correlation between T2 values and Mankin scores. The Mankin scores are weakly positively correlated with T2 ( $\mathrm{r}=0.337, \mathrm{P}=0.004)$, markedly negatively correlated with $\mathrm{T} 2 *(\mathrm{r}=-0.501, \mathrm{P}<0.001)$, and strongly negatively correlated with MTR $(\mathrm{r}=-0.678, \mathrm{P}<0.001)$. MTR, magnetization transfer ratio.

conventional sequences. Although conventional MTR can be used to study macromolecular proton pools indirectly, conventional clinical sequences are limited in acquiring the adequate signal from deep cartilage with short mean transverse relaxation times. On the other hand, the MTR of the UTE-MT sequence allows the acquisition and quantification of this otherwise "invisible" tissue component. As a result, UTE-MT imaging may provide a complete picture of cartilage matrix changes in terms of osteoarthritic cartilage compared with that provided by conventional MT imaging, which can only detect surface cartilage signal. Quantitative UTE-MT may thus hold considerable potential as a new diagnostic technique for the evaluation of cartilage degeneration.

The quality of UTE T2* mapping for assessing articular cartilage has been verified by many research teams. Although there are a few studies that did not find $\mathrm{T}^{*}$ mapping to be particularly useful in grading cartilage degeneration $(22,53)$, these studies used $\mathrm{T}^{*}$ mapping based on fast-field echo (FFE) multi-echo gradient echo which is different from the technique presented in this study. Our previous experiments showed that UTE-T2* mapping was less sensitive to the magic angle effect and more sensitive to deep cartilage, making it a satisfactory method for evaluating the quality of cartilage (23). A recent clinical study suggested that decreasing the UTE-T2* profile slopes reflected disruption in the collagenous matrix of deep cartilage in a population at increased risk for OA (28). Our present study showed that UTE-T2* values decreased as the histopathologic grades increased, a result consistent with previous studies (54). In addition, previous research (33) (UTE-T2*mapping via bi-component analysis) found that as the percentage of short $\mathrm{T} 2^{*}$ components increased (short T2* water), the UTE-T2* values decreased and the grade of cartilage degeneration increased. Given that PG and collagen contents are reduced during cartilage degeneration, the short $\mathrm{T} 2{ }^{*}$ water associated with these components should decrease accordingly. At the same time, 
Group1 vs. Group2

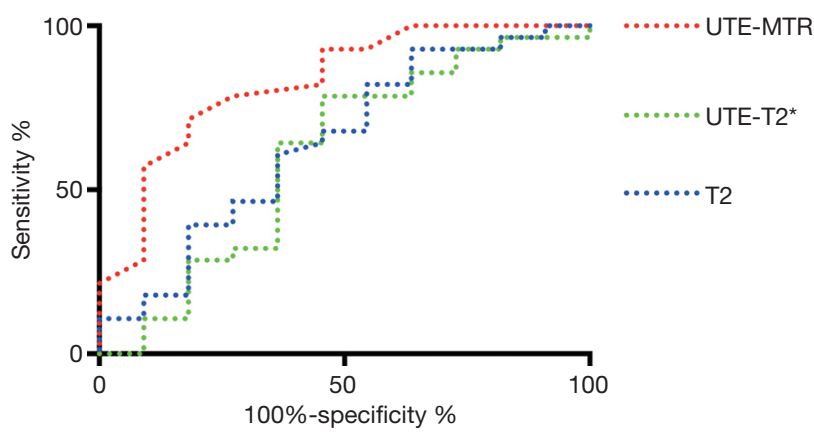

Figure 5 Receiver operating characteristic (ROC) curves of three MR imaging techniques for the diagnosis of early degeneration of cartilage. The areas under the curve (AUC) of UTE-MTR (AUC $=0.828, \mathrm{P}=0.002)$ are significantly higher than those of UTE T2* $(\mathrm{AUC}=0.604, \mathrm{P}=0.318)$ and $\mathrm{T} 2$ mapping $(\mathrm{AUC}=0.644, \mathrm{P}=0.165)$ at 0.05 significance level. UTE-MTR, ultrashort echo time magnetization transfer ratio.

the collagen fiber network structure is destroyed, increasing the surface area of exposed collagen fiber. As a result, the short $\mathrm{T}^{*}$ water combined with this exposed collagen fiber consequently increases. When the newly bound water increase is more significant than that of the combined PG and collagen combined water loss, the total $\mathrm{T} 2{ }^{*}$ value decreases. This pathological alteration may explain the nonsignificant differences in UTE single-component T2* values between normal cartilage and early degeneration of cartilage. The loss of collagen and PG combined with the increase of newly bound water are similar in early degeneration of cartilage; their aggregate effect may have a non-significant impact on total UTE-T2* values.

T2 mapping has been used widely and has proven to be important in the evaluation of cartilage.

David-Vaudey et al. showed that T2 relaxation time reflected OA pathogenesis via a positive correlation with a histologic grading scale and that $\mathrm{T} 2$ relaxation time was affected by collagen matrix disarrangement and water increase in cartilage (37). The correlation between the T2 values and the relative water content of articular cartilage was also reported in a rat OA model $(42,43)$. One previous study in dogs (44) found a strong positive correlation $(\mathrm{r}=0.758, \mathrm{P}<0.01)$ between $\mathrm{T} 2$ relaxation times and Mankin scores in dogs that had a large subchondral bone defect created in the proximal tibial metaphysis. However, another study in dogs showed only a weakly negative correlation between the T2 mapping and PG content $(\mathrm{r}=-0.32, \mathrm{P}<0.05)$ (45). Weak or non-existent correlations between T2 mapping and cartilage assessments are not scarce. Regatte et al. (38) reported that the range of $\mathrm{T} 2$ values varied from $32 \pm 2$ to $45 \pm 4 \mathrm{~ms}$ and increased with different grades of OA; however, no great differences between OA groups were demonstrated. Kim et al. (53) showed that T2 mapping was correlated with histological degeneration, but the strength of the correlation was weak compared with histopathologic grades $(r=0.313, P<0.05)$. A statistically significant difference in mean $\mathrm{T} 2$ relaxation value was observed only between the normal and severe groups. We obtained similar results in the present study. The low correlation between T2 values and Mankin scores may reflect a considerable flaw in evaluating cartilage quality when using T2 mapping. This result may be due to the susceptibility of T2 mapping to the magic angle effect and to the effects of different components in the cartilage during the occurrence and development of OA $(15,55)$.

The UTE-MTR values showed significant differences between the healthy group and mild degeneration group and were also highly correlated with histopathologic grades, suggesting that significant macromolecular loss may begin during early OA and that UTE-MTR values of cartilage are sensitive to changes in macromolecular components during early degeneration. Articular cartilage is a dense avascular connective tissue consisting of relatively few cells and a highly charged and hydrated extracellular matrix (ECM), composed of collagen, PGs, and water (3). The very early stage of cartilage injury is mainly due to the loss of PG and collagen in the cartilage matrix. MTR value is mainly correlated with collagen and PG content, but T2/T2* value is proportionally correlated with collagen orientation and water content. Therefore, UTE-MTR values may be highly sensitive in detecting early cartilage degeneration, making it a superior method over UTE single-component T2* analysis and $\mathrm{T} 2$ mapping.

There are several limitations in our current study. First, the sample size was small, although it was indirectly increased by drawing 4 ROIs on each specimen. More specimens will be included in our future study. Second, this study was performed ex vivo (at room temperature, extracellular $\mathrm{pH}$ ), and the measurement condition was different from the real physiological environment. This is an expected limitation associated with such an $e x$ vivo study before eventual in vivo translation. A comprehensive evaluation of cartilage will be investigated further in later studies. Another limitation was the potential effect of 
necessary specimen rehydration on MTR values. Fishbein et al. reported that MTR values of in vitro cartilage decreased substantially during immersion in aqueous solutions, because of increased hydration upon direct exposure of all cartilage surfaces to the aqueous solution, including the transitional and deep zones (56). However, we mitigated these effects by immersion in perfluorocarbon liquids (Fomblin), as detailed in similar studies $(56,57)$. Additionally, this study could not utilize quantitative biochemical measurements for PG and collagen of cartilage, as none were available. Therefore, the quantitative MR data obtained from the experiment have not been quantitatively confirmed using the corresponding biochemical components of cartilage. Another limitation is that MTR values are susceptible to many factors, including deviation frequencies, radiofrequency angles, and field intensities. All these factors may result in small discrepancies of MTR values, thereby limiting its application in cartilage degeneration evaluation. Our next study will address this by quantitatively analyzing the biochemical components of cartilage and in vivo tests. Finally, Mankin scores were used to evaluate the changes in morphological and histochemical characteristics; cartilage volume and bone were not included. These limitations notwithstanding, the UTE sequence can provide information about the subchondral bone.

\section{Conclusions}

Our preliminary result indicated that UTE-MTR values were strongly correlated with histological grades (Mankin scores) of cartilage degeneration and that diagnostic efficacy was better than both UTE T2* mapping and T2 mapping in detecting early cartilage degeneration. Once the clinical potential of this technique has been confirmed, UTEMT may provide a promising imaging biomarker with potential application in a more comprehensive diagnosis and monitoring of cartilage degeneration.

\section{Acknowledgments}

Funding: This study was supported by the grant from the National Natural Science Foundation of China (No. 81871325,81801656 ), the National Institutes of Health (1R01 NS092650), the Shanghai Science and Technology Commission, the International Cooperation and Exchange Project (16410722200), the Shanghai Pujiang Program (17PJ1407800), and the Incubation Program of the National Natural Science Foundation of China from
Shanghai Tenth People's Hospital of Tongji University (SYGZRPY2017039).

\section{Footnote}

Conflicts of Interest: The authors have no conflicts of interest to declare.

Ethical Statement: The study was approved by the Ethics Committee at the Shanghai Tenth People's Hospital of Tongji University (Ethics Committee approval number: shsy-iec-ky-3964), and written informed consent was obtained from all patients.

\section{References}

1. Roemer FW, Crema MD, Trattnig S, Guermazi A. Advances in imaging of osteoarthritis and cartilage. Radiology 2011;260:332-54.

2. Atukorala I, Kwoh CK, Guermazi A, Roemer FW, Boudreau RM, Hannon MJ, Hunter DJ. Synovitis in knee osteoarthritis: a precursor of disease? Ann Rheum Dis 2016;75:390-5.

3. Waldschmidt JG, Rilling RJ, Kajdacsy-Balla AA, Boynton $M D$, Erickson SJ. In vitro and in vivo MR imaging of hyaline cartilage: zonal anatomy, imaging pitfalls, and pathologic conditions. Radiographics 1997;17:1387-402.

4. Jurvelin J, Kiviranta I, Saamanen AM, Tammi M, Helminen HJ. Partial restoration of immobilizationinduced softening of canine articular cartilage after remobilization of the knee (stifle) joint. J Orthop Res 1989;7:352-8.

5. Palmer AJ, Brown CP, McNally EG, Price AJ, Tracey I, Jezzard P, Carr AJ, Glyn-Jones S. Non-invasive imaging of cartilage in early osteoarthritis. Bone Joint J 2013;95B:738-46.

6. Favero M, Ramonda R, Goldring MB, Goldring SR, Punzi L. Early knee osteoarthritis. RMD Open 2015;1:e000062.

7. Lange T, Knowles BR, Herbst M, Izadpanah K, Zaitsev M. Comparative T2 and T1rho mapping of patellofemoral cartilage under in situ mechanical loading with prospective motion correction. J Magn Reson Imaging 2017;46:452-60.

8. Neu CP. Functional imaging in OA: role of imaging in the evaluation of tissue biomechanics. Osteoarthritis Cartilage 2014;22:1349-59.

9. Nishioka H, Hirose J, Nakamura E, Oniki Y, Takada K, Yamashita Y, Mizuta H. T1rho and T2 mapping reveal the in vivo extracellular matrix of articular cartilage. J Magn 
Reson Imaging 2012;35:147-55.

10. Surowiec RK, Lucas EP, Ho CP. Quantitative MRI in the evaluation of articular cartilage health: reproducibility and variability with a focus on T2 mapping. Knee Surg Sports Traumatol Arthrosc 2014;22:1385-95.

11. Tsushima H, Okazaki K, Takayama Y, Hatakenaka M, Honda H, Izawa T, Nakashima Y, Yamada H, Iwamoto Y. Evaluation of cartilage degradation in arthritis using T1rho magnetic resonance imaging mapping. Rheumatol Int 2012;32:2867-75.

12. Li X, Cheng J, Lin K, Saadat E, Bolbos RI, Jobke B, Ries MD, Horvai A, Link TM, Majumdar S. Quantitative MRI using T1rho and T2 in human osteoarthritic cartilage specimens: correlation with biochemical measurements and histology. Magn Reson Imaging 2011;29:324-34.

13. Truhn D, Sondern B, Oehrl S, Tingart M, Knobe M, Merhof D, Kuhl C, Thuring J, Nebelung S. Differentiation of human cartilage degeneration by functional MRI mapping-an ex vivo study. Eur Radiol 2019;29:6671-81.

14. Thüring J, Linka K, Itskov M, Knobe M, Hitpass L, Kuhl C, Truhn D, Nebelung S. Multiparametric MRI and Computational Modelling in the Assessment of Human Articular Cartilage Properties: A Comprehensive Approach. Biomed Res Int 2018;2018:9460456.

15. Linka K, Itskov $M$, Truhn D, Nebelung S, Thuring J. T2 MR imaging vs. computational modeling of human articular cartilage tissue functionality. J Mech Behav Biomed Mater 2017;74:477-87.

16. Wan C, Ge L, Souza RB, Tang SY, Alliston T, Hao Z, Li X. T1rho-based fibril-reinforced poroviscoelastic constitutive relation of human articular cartilage using inverse finite element technology. Quant Imaging Med Surg 2019;9:359-70.

17. Xia Y, Moody JB, Alhadlaq H. Orientational dependence of T2 relaxation in articular cartilage: A microscopic MRI (microMRI) study. Magn Reson Med 2002;48:460-9.

18. Xia Y, Moody JB, Burton-Wurster N, Lust G. Quantitative in situ correlation between microscopic MRI and polarized light microscopy studies of articular cartilage. Osteoarthritis Cartilage 2001;9:393-406.

19. Wheaton AJ, Casey FL, Gougoutas AJ, Dodge GR, Borthakur A, Lonner JH, Schumacher HR, Reddy R. Correlation of T1rho with fixed charge density in cartilage. J Magn Reson Imaging 2004;20:519-25.

20. Li X, Han ET, Ma CB, Link TM, Newitt DC, Majumdar $\mathrm{S}$. In vivo $3 \mathrm{~T}$ spiral imaging based multi-slice $\mathrm{T}(1 \mathrm{rho})$ mapping of knee cartilage in osteoarthritis. Magn Reson
Med 2005;54:929-36.

21. van Tiel J, Kotek G, Reijman M, Bos PK, Bron EE, Klein S, Nasserinejad K, van Osch GJ, Verhaar JA, Krestin GP, Weinans H, Oei EH. Is T1rho Mapping an Alternative to Delayed Gadolinium-enhanced MR Imaging of Cartilage in the Assessment of Sulphated Glycosaminoglycan Content in Human Osteoarthritic Knees? An in Vivo Validation Study. Radiology 2016;279:523-31.

22. Nebelung S, Brill N, Tingart M, Pufe T, Kuhl C, Jahr H, Truhn D. Quantitative OCT and MRI biomarkers for the differentiation of cartilage degeneration. Skeletal Radiol 2016;45:505-16.

23. Shao H, Pauli C, Li S, Ma Y, Tadros AS, Kavanaugh A, Chang EY, Tang G, Du J. Magic angle effect plays a major role in both T1rho and T2 relaxation in articular cartilage. Osteoarthritis Cartilage 2017;25:2022-30.

24. Li X, Pai A, Blumenkrantz G, Carballido-Gamio J, Link T, Ma B, Ries M, Majumdar S. Spatial distribution and relationship of $\mathrm{T} 1$ rho and $\mathrm{T} 2$ relaxation times in knee cartilage with osteoarthritis. Magn Reson Med 2009;61:1310-8.

25. Chang EY, Du J, Bae WC, Chung CB. Qualitative and Quantitative Ultrashort Echo Time Imaging of Musculoskeletal Tissues. Semin Musculoskelet Radiol 2015;19:375-86.

26. Muir P, McCarthy J, Radtke CL, Markel MD, Santschi EM, Scollay MC, Kalscheur VL. Role of endochondral ossification of articular cartilage and functional adaptation of the subchondral plate in the development of fatigue microcracking of joints. Bone 2006;38:342-9.

27. Salim AS. Percutaneous decompression and irrigation for large bowel obstruction. New approach. Dis Colon Rectum 1991;34:973-80; discussion 978-80.

28. Williams AA, Titchenal MR, Andriacchi TP, Chu CR. MRI UTE-T2* profile characteristics correlate to walking mechanics and patient reported outcomes 2 years after ACL reconstruction. Osteoarthritis Cartilage 2018;26:569-79.

29. Du J, Takahashi AM, Chung CB. Ultrashort TE spectroscopic imaging (UTESI): application to the imaging of short T2 relaxation tissues in the musculoskeletal system. J Magn Reson Imaging 2009;29:412-21.

30. Shao H, Chang EY, Pauli C, Zanganeh S, Bae W, Chung CB, Tang G, Du J. UTE bi-component analysis of T2* relaxation in articular cartilage. Osteoarthritis Cartilage 2016;24:364-73.

31. Ma YJ, Shao H, Du J, Chang EY. Ultrashort echo time magnetization transfer (UTE-MT) imaging and modeling: magic angle independent biomarkers of tissue properties. 
NMR Biomed 2016;29:1546-52.

32. High RA, Ji Y, Ma YJ, Tang Q, Murphy ME, Du J, Chang EY. In vivo assessment of extracellular $\mathrm{pH}$ of joint tissues using acidoCEST-UTE MRI. Quant Imaging Med Surg 2019;9:1664-73.

33. Pauli C, Bae WC, Lee M, Lotz M, Bydder GM, D'Lima DL, Chung CB, Du J. Ultrashort-echo time MR imaging of the patella with bicomponent analysis: correlation with histopathologic and polarized light microscopic findings. Radiology 2012;264:484-93.

34. Song JX, Lu JH, Liu LF, Chen LL, Durairajan SS, Yue Z, Zhang HQ, Lia M. B1 is involved in autophagy inhibition caused by SNCA/a-synuclein overexpression: a process modulated by the natural autophagy inducer corynoxine B. Autophagy 2015;11:1708.

35. Mankin HJ. Biochemical and metabolic aspects of osteoarthritis. Orthop Clin North Am 1971;2:19-31.

36. Dunn TC, Lu Y, Jin H, Ries MD, Majumdar S. T2 relaxation time of cartilage at $\mathrm{MR}$ imaging: comparison with severity of knee osteoarthritis. Radiology 2004;232:592-8.

37. David-Vaudey E, Ghosh S, Ries M, Majumdar S. T2 relaxation time measurements in osteoarthritis. Magn Reson Imaging 2004;22:673-82.

38. Regatte RR, Akella SV, Lonner JH, Kneeland JB, Reddy R. T1rho relaxation mapping in human osteoarthritis (OA) cartilage: comparison of T1rho with T2. J Magn Reson Imaging 2006;23:547-53.

39. Apprich S, Mamisch TC, Welsch GH, Stelzeneder D, Albers C, Totzke U, Trattnig S. Quantitative T2 mapping of the patella at $3.0 \mathrm{~T}$ is sensitive to early cartilage degeneration, but also to loading of the knee. Eur J Radiol 2012;81:e438-43.

40. Kwack KS, Min BH, Cho JH, Kim JM, Yoon SH, Kim SY. T2 relaxation time mapping of proximal tibiofibular cartilage by 3 -tesla magnetic resonance imaging. Acta Radiol 2009;50:1049-56.

41. Kijowski R, Blankenbaker DG, Munoz Del Rio A, Baer GS, Graf BK. Evaluation of the articular cartilage of the knee joint: value of adding a T2 mapping sequence to a routine MR imaging protocol. Radiology 2013;267:503-13.

42. Chou MC, Tsai PH, Huang GS, Lee HS, Lee CH, Lin MH, Lin CY, Chung HW. Correlation between the MR $\mathrm{T} 2$ value at $4.7 \mathrm{~T}$ and relative water content in articular cartilage in experimental osteoarthritis induced by ACL transection. Osteoarthritis Cartilage 2009;17:441-7.

43. Ali TS, Prasadam I, Xiao Y, Momot KI. Progression of Post-Traumatic Osteoarthritis in rat meniscectomy models: Comprehensive monitoring using MRI. Sci Rep 2018;8:6861.

44. Yan D, Liu TX, Liu BY, Wang L, Qian ZH, Cheng XG, Li KC. Effects of Structural Changes in Subchondral Bone on Articular Cartilage in a Beagle Dog Model. Biomed Environ Sci 2017;30:194-203.

45. Franklin SP, Stoker AM, Lin ASP, Pownder SL, Burke EE, Bozynski CC, Kuroki K, Guldberg RE, Cook JL, Holmes SP. T1 $\rho$, T2 mapping, and EPIC- $\mu$ CT Imaging in a Canine Model of Knee Osteochondral Injury. J Orthop Res 2019. [Epub ahead of print].

46. Ellermann J, Ling W, Nissi MJ, Arendt E, Carlson CS, Garwood M, Michaeli S, Mangia S. MRI rotating frame relaxation measurements for articular cartilage assessment. Magn Reson Imaging 2013;31:1537-43.

47. Gray ML, Burstein D, Lesperance LM, Gehrke L. Magnetization transfer in cartilage and its constituent macromolecules. Magn Reson Med 1995;34:319-25.

48. Lattanzio PJ, Marshall KW, Damyanovich AZ, Peemoeller H. Macromolecule and water magnetization exchange modeling in articular cartilage. Magn Reson Med 2000;44:840-51.

49. Zuo H, Yao W, Qu N, Yang S, Wang J, Cui X. Quantitative evaluation in combination with nonquantitative evaluation in early patellar cartilage osteoarthritis at $3.0 \mathrm{~T}$. Clin Interv Aging 2014;9:1133-43.

50. Welsch GH, Trattnig S, Scheffler K, Szomonanyi P, Quirbach S, Marlovits S, Domayer S, Bieri O, Mamisch TC. Magnetization transfer contrast and T2 mapping in the evaluation of cartilage repair tissue with $3 \mathrm{~T}$ MRI. J Magn Reson Imaging 2008;28:979-86.

51. Henkelman RM, Stanisz GJ, Kim JK, Bronskill MJ. Anisotropy of NMR properties of tissues. Magn Reson Med 1994;32:592-601.

52. Nissi MJ, Salo EN, Tiitu V, Liimatainen T, Michaeli S, Mangia S, Ellermann J, Nieminen MT. Multi-parametric MRI characterization of enzymatically degraded articular cartilage. J Orthop Res 2016;34:1111-20.

53. Kim T, Min BH, Yoon SH, Kim H, Park S, Lee HY, Kwack KS. An in vitro comparative study of T2 and T2* mappings of human articular cartilage at 3-Tesla MRI using histology as the standard of reference. Skeletal Radiol 2014;43:947-54.

54. Williams A, Qian Y, Bear D, Chu CR. Assessing degeneration of human articular cartilage with ultra-short echo time (UTE) T2* mapping. Osteoarthritis Cartilage 2010;18:539-46.

55. Mlynárik V, Szomolanyi P, Toffanin R, Vittur F, Trattnig S. 
Transverse relaxation mechanisms in articular cartilage. J Magn Reson 2004;169:300-7.

56. Fishbein KW, Sexton KW, Celik H, Reiter DA, Bouhrara M, Spencer RG. Stabilization of T2 relaxation and magnetization transfer in cartilage explants by immersion in perfluorocarbon liquid. Magn Reson Med

Cite this article as: Yang J, Shao H, Ma Y, Wan L, Zhang Y, Jiang J, Du J, Tang G. Quantitative ultrashort echo time magnetization transfer (UTE-MT) for diagnosis of early cartilage degeneration: comparison with UTE-T2* and T2 mapping. Quant Imaging Med Surg 2020;10(1):171-183. doi: 10.21037/qims.2019.12.04
2019;81:3209-17.

57. Chang EY, Du J, Bae WC, Statum S, Chung CB. Effects of Achilles tendon immersion in saline and perfluorochemicals on T2 and T2*. J Magn Reson Imaging 2014;40:496-500. 